\title{
PAFNútiY LVóvitch TCHEbySheV E O NASCIMENTO DA ESCOLA RUSSA EM TEORIA DOS NÚMEROS
}

\author{
Bernadete Morey \\ Universidade Federal do Rio Grande do Norte - UFRN - Brasil \\ Adgam Sultanov \\ Universidade Estatal de Penza - UEP - Rússia
}

(aceito para publicação em novembro de 2016)

\begin{abstract}
Resumo
O artigo tem o objetivo de apresentar a pessoa e a obra de Pafnútiy Lvóvitch Tchebyshev aliada à sua importante contribuição no desenvolvimento da matemática e na formação de uma escola russa em Teoria dos Números.

Palavras-chave: História da Álgebra, História da Teoria dos Números, Tchebyshev.
\end{abstract}

\section{[PAFNUTIY LVOVICH CHEBYSHEV AND THE BIRTH OF THE NUMBER THEORY RUSSIAN SCHOOL]}

\begin{abstract}
The purpose of this paper is to present the life and work of the Russian mathematician Pafnutiy Lvovich Chebyshev whose work contributed to the development of Mathematics and the birth of the Russian tradition in Number Theory.
\end{abstract}

Keywords: History of Algebra, History of Number Theory, Chebyshev.

\section{[П. Л. ЧЕБЫШЕВ И РОЖДЕНИЕ РУССКОЙ ШКОЛЫ ТЕОРИИ ЧИСЕЛ]}




\section{АННотАцИЯ}

Статья представляет читателю жизнь и творчество Пафнутя Лвовича Чебышева, его роль в развитии математики а так же в становлении русской школы теории чисел.

Ключевые слова: История Алгебры, История Теории Чисел, Чебышев.

\section{Introdução}

É possível que já tenhamos ouvido falar em Tchebyshev ligado à Estatística com a Lei dos Grandes Números ou ainda ligado à Análise com o Teorema de Tchebyshev sobre a integral do binômio. Propomos neste artigo conhecer este matemático (e mecânico) por meio de sua ligação à Teoria dos Números. Neste artigo nos propomos a examinar a trajetória do menino nascido numa região próxima a Moscou no início do século XIX, trajetória esta que o levou a ter seu nome conhecido em diferentes áreas da matemática. Aqui neste artigo trataremos mais especificamente de sua atuação em Teoria dos Números.

Leonhard Euler (1707-1783) em São Petersburgo contribuiu enormemente para o desenvolvimento da teoria dos números. Ele generalizou o resultado de Fermat para o caso divisibilidade por números compostos, criou a teoria geral dos chamados resíduos exponenciais, obteve um grande número de resultados distintos sobre a representação de números sob a forma de terminado tipo. Investigou também uma série de sistemas de equações indefinidas e obteve resultados sobre a partição de números em parcelas. Depois dos trabalhos de Euler, Lagrange (1735 - 1813) e Legendre (1753 - 1833) obtiveram resultados que juntamente com os de Euler criaram a base para uma teoria completa que recebeu mais tarde com Gauss o nome de teoria das congruências. Os trabalhos de Gauss em teoria das congruências de $2^{\circ}$. grau lhe conferiram um caráter de finalização tal que atualmente toda esta área da teoria dos números se baseia em resultados expostos na obra Disquisitiones arithmeticae (Bukhshtab, p.11).

No entanto, o nascimento de uma escola de estudiosos russos em teoria dos números no sentido próprio do termo se dá com Tchebyshev. Tal escola, a escola de São Peterburgo em teoria dos números ${ }^{1}$ fez surgir grandes nomes. Além do próprio Tchebyshev podemos citar Korkin, Zolotariev, Markov, Voronoy. É devido ao seu papel de iniciador que elegemos Tchebyshev como figura central neste artigo. A respeito dos demais membros desta escola, falaremos algumas palavras na conclusão do presente artigo.

\section{Breve biografia}

\footnotetext{
${ }^{1}$ Em 1947, no centenário da chegada de Tchebyshev a São Petersburgo, publicou-se um livro chamado Петербургская школа теории чисел (A escola de São Petersburgo em Teoria dos Números) que traz a biografia e os principais trabalhos comentados dos nomes acima citados. O livro foi traduzido para o ingles e publicado conjuntamente pela American Mathematical Society e London Mathematical Society em 2005.
} 
Pafnútiy Lvóvitch Tchebyshev ${ }^{2}$ nasceu em 26 (14) de maio de $1821^{3}$ na propriedade de seu pai, na aldeia Okátov na região de Kaluga ${ }^{4}$ a sudoeste de Moscou. O pequeno Pafnútiy nasceu numa família pertencente a um ramo antigo da nobreza e seu pai, homem bem-educado, gozava de riqueza material razoável. O pequeno Pafnútiy recebeu sua educação inicial em casa: sua mãe, Agrafena Lvóvna, lhe ensinou as primeiras letras enquanto que sua prima Audótia Kintiliánovna Sukhariôva, moça educada e que também exercia o papel de governanta na casa dos Tchebyshev, ensinou-lhe aritmética e línguas estrangeiras (Prúdnikov, p.18).

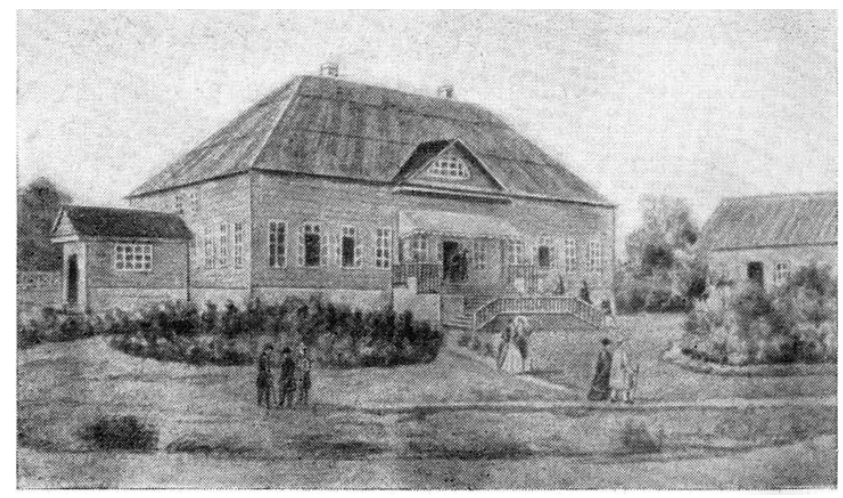

Figura 1. Casa dos Tchebyshev em Okátov. Fonte: Prúdnikov, 1976, p. 30

Em 1832 os Tchebyshev mudaram-se para Moscou, indo morar na casa da qual eram proprietários. O objetivo da mudança era preparar os meninos (Pafnútiy e seu irmão mais velho, Pável) para entrada na universidade. Contratou-se para a tarefa de preparação os melhores professores de Moscou e foi neste período que se notou o talento do jovem para a matemática, talento este que o levou à opção de entrar na faculdade de física e matemática da Universidade de Moscou $^{5}$ (Bernshtein, 1944, p. 6). Entrou na universidade em 1837 e logo no final do primeiro ano despertou a atenção de Nikolái Dmítrievitch Brashman (17961866) que o incentivou a dedicar-se exclusivamente à matemática; terminou o curso universitário (bacharelado) na Universidade de Moscou em 1841.

Em 1840 a fome assolou boa parte da Rússia e muitos dos homens abastados perderam suas posses. O pai de Pafnútiy foi um deles e a família teve de se mudar para a

2 Tchebyshev, assim como a maioria dos nomes próprios russos, pode ser transliterado de várias maneiras. No presente artigo adotou-se a transliteração dos nomes próprios russos que (na opinião da primeira autora do artigo) soasse em português o mais próximo possível do original russo. É possível então que tal grafia se diferencie da que se constuma encontrar nos textos traduzidos ao português, que via de regra, são traduzidos do inglês ou do francês. ${ }^{3}$ A Rússia adotou o calendário gregoriano em fevereiro de 1918. É comum, então que nos textos históricos apareçam duas datas indicando o evento no calendário juliano e no gregoriano.

${ }^{4}$ Kaluga, cidade/centro administrativo da Região de Kaluga (antiga Gubêrnya de Kaluga) fica a 180 km a sudoeste de Moscou.

${ }^{5}$ Fundada em 1755, por ordem da imperatriz Elizabeta, que reinou imediatamente após Pedro, o Grande.

RBHM, Vol. 16, n 32, p. 19-31, 2016 
aldeia. Em Moscou, na casa da família, ficaram somente o jovem Pafnútiy e dois de seus irmãos. A situação financeira dos jovens Tchebyshev não era boa, pois, seu pai não podia mais apoiá-los financeiramente, deixando-lhe apenas a casa para morar. Mesmo assim, o jovem preferiu se aplicar à continuidade de sua carreira científica do que buscar um posto de trabalho. Em 8 (20) de junho de 1846, em sessão pública, Tchebyshev defendeu a dissertação Experiência de análise elementar da teoria das probabilidades, recebendo, assim, o título de mestre.

Em 1847 Tchebyshev mudou-se para São Petersburgo em cuja universidade defendeu a dissertação intitulada Sobre integração por meio de logaritmos, defesa esta que lhe deu o direito de lecionar na dita universidade.

$\mathrm{Na}$ época em que Tchebyshev entrou para a Universidade de São Petersburgo, entrou também Viktor Yakóvlevitch Bunyakóvsky um matemático já conhecido e membro da Academia de Ciências. Na cátedra de matemática aplicada já estava Iócif Ivánovitch Sómov e com essas duas pessoas Tchebyshev manteve laços de amizade durante toda a vida. (DELONE, 1947, p.8).

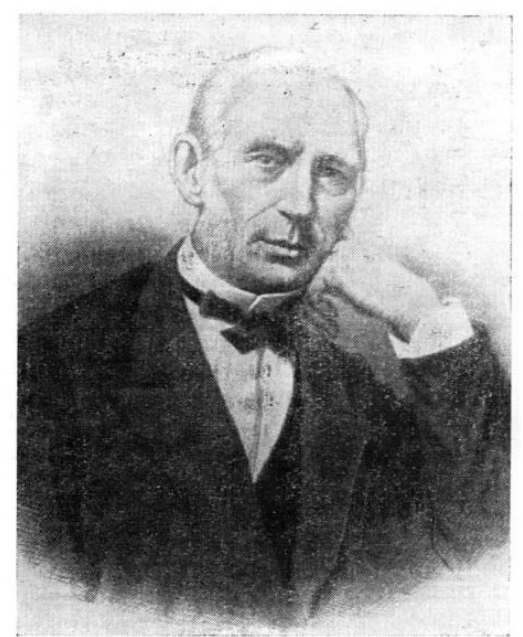

Figura 2. Tchebyshev (anos 70 do século XIX) Fonte: Prúdnikov, 1976, p.78

A atividade de Tchebyshev na academia de ciências começou quase ao mesmo tempo em iniciou o seu trabalho na universidade. Prúdnikov nos conta que em 1844 Pável Nikoláievitch Fuss (1798-1855)

\footnotetext{
${ }^{6}$ Nikolay Fuss (1755 - 1825), pai de Pável Nikoláievitch Fuss mudou-se da Suissa para São Petersburgo aos 17 anos para assumir a função de secretário de Leonhard Euler. Na verdade, além de secretário também foi aluno de
} 
Euler, encontrou nos arquivos da academia alguns escritos de seu bisavô, parte deles em teoria dos números. A academia decidiu publicá-los e incumbiu Bunyakóvsky de sistematizálos. Bunyakóvsky por sua vez convidou Tchebyshev como auxiliar. Juntos eles elaboraram o índice sistemático que mais tarde foi publicado no primeiro tomo de Leonardi euleri commentationes arithmeticae collecta em São Petersburgo em 1849. (PRÚDNIKOV, 1976, p.71)

No ano letivo seguinte (1848/1849) a teoria dos números entrou no rol de disciplinas que Tchebyshev deveria lecionar. Ele assumiu a tarefa com muito interesse e a necessidade de expor a disciplina para uma plateia de estudantes o estimulou a fazer suas próprias investigações em teoria dos números. Tudo isto o levou a solicitar ao conselho universitário, em novembro de 1848, que examinasse sua composição sobre teoria das congruências como dissertação de doutorado. O conselho acatou a solicitação e, em 15 (27) de maio de 1849 Tchebyshev defende a dissertação pela qual recebe o título de doutor, denominada Teoria das congruências. (PRÚDNIKOV, 1976, p.72)

Tchebyshev exerceu a docência em São Petersburgo por 35 anos, de 1847 a 1882. Lecionou geometria analítica, álgebra abstrata, teoria dos números, calculo integral, teoria das probabilidades, teoria das diferenças finitas, teoria da funções elípticas e teoria da integral definida.

Bernshtein (1944, p.7) nos conta que dos poucos amigos que tinha, Tchebyshev gostava especialmente de visitar um deles: Iócif Ivánovitch Sómov ${ }^{7}$ (1815-1876), membro da academia de Ciências ${ }^{8}$. Em suas longas conversas, Tchebyshev se interessava em saber se suas descobertas já tinham sido precedidas por alguém. O fato do acadêmico ter vasta leitura e estar sempre a par do que se produzia em matemática facilitava em muito o trabalho de Tchebyshev. Com a maturidade científica Tchebyshev foi se adentrando cada vez mais em áreas pouco exploradas, de modo que se tornava cada vez menos provável que os resultados a que chegava já tivessem sido obtidos por outros. Ele estudava profundamente os grandes matemáticos: Euler, Lagrange, Abel, Gauss, mas gastava pouco tempo se inteirando do que estava sendo produzido pelos seus contemporâneos. Dizia que se aprofundar muito no que outros matemáticos estavam fazendo podia distraí-lo do seu próprio trabalho.

Ele continuava a orientar seus alunos mesmo depois destes terem terminado o curso universitário. Uma vez por semana, em hora determinada, ele recebia em sua casa qualquer um que quisesse falar sobre seus próprios estudos matemáticos e receber alguma orientação. Raramente alguém saia sem ter recebido novas ideias e incentivo ao novo trabalho.

Euler terminando por se tornar um matemático conhecido. Casou-se com Albertine Benediktine Phillipine Louise neta do grande matemático. Um dos quatro filhos homens de Nikolay Fuss foi Pável Nikoláeivitch Fuss que foi aluno da Academia de São Petersburgo e que em 1826 substituiu seu pai no posto de secretário da Academia. No início de sua carreira P. N. Fuss publicou alguns de trabalhos ciêntíficos de caráter independente, mas, posteriormente todo seu trabalho na Academia foi de caráter bibliográfico e relacionado às composições de seu bisavô Leonhard Euler. Das edições críticas de Fuss sobre composições de Euler apresentadas na Academia, com excessão da primeira, todas foram de publicadas conjuntamente com Bunyakovsky.

${ }^{7}$ Sómov tinha sido orientado na Universidade de Moscou por Brashman, o mesmo orientador de Tchebyshev.

${ }^{8}$ Fundada em 1724 por rdem do imperador Pedro I (Pedro, o Grande). 
Em relação aos seus últimos dias, cito Bernshtein (1944, p.9):

“Em relação aos últimos dias da vida de P. L. Tchebyshev sabe-se apenas que a alguns dias de sua morte ele adoeceu de uma forma leve de influenza e, apesar de não se sentir bem, não ficou de cama. Na véspera de sua morte recebeu visitas, como era de seu costume e ninguém poderia imaginar que seu fim estava assim tão proximo. Na manhã de 8 de dezembro (24 de novembro) de 1894 Pafnútiy Lvóvitch, sentado à escrivaninha e tomando uma chícara de chá, sentiu-se mal de repente e depois de uma breve agonia, faleceu de parada cardíaca”. (BERNSHTEIN, 1944, p. 9) (tradução nossa)

\section{Teoria dos números anterior de Tchebyshev}

Tchebyshev tinha um espectro de interesses bastanto amplo; ele publicou uma série de trabalhos em teoria das probabilides, análise matemática e teoria dos mecanismos. No entanto, o escopo deste artigo nos leva a nos restringir mais propiamente à teoria dos números.

Na breve exposição que faremos a seguir sobre o desenvolvimento da teoria dos números antes de Tchebyshev seguiremos Bukhshtab (1966, p.10).

Os trabalhos de Euclides atribuem pouco espaço às questões teorico-numéricas mas ali encontramos estudos sobre divisibilidade e o resultado tão simples quanto fundamental sobre a infinitude dos números primos. Além disso, o chamado Crivo de Eratóstenes era conhecidos dos matemáticos gregos. Mais tarde Diofanto debruçou-se sobre uma série de problemas sobre a representação de números sob determinada forma e sobre questões mais gerais de resolução de equações indeterminadas em números racionais positivos. Mais tarde esses problemas servirão de ponto de partida para a teoria das formas e da teoria das equações diofantinas.

Os matemáticos do Oriente Próximo e Médio, ao cultivar sua própria cultura matemática dedicaram-se principalmente à álgebra e trigonometria, demonstrando pouco interesse nos problemas teorico-numéricos. A Europa anterior ao século XVII pouco se dedicou ao estudos de métodos gerais de resolução. O maior legado para a teoria dos números desta época foi deixado por Leonardo de Pisa, o Fibonacci (? - 1250) e por Regiomontano (1436 - 1476) que ao ter contato com os trabalhos de Diofanto ${ }^{9}$ passou a estudá-los sistematicamente.

No século XVI e início do século XVII na Europa foram publicados em francês e em latim textos de Diofanto que receberam a atenção de matemáticos europeus daquele tempo. Sabe-se que Viète (1540 - 1603) e Claude Gaspard Bachet de Méziriac (1581 - 1638) se debruçaram sobre estes textos elaborando comentários e completando-os com novos resultados.

\footnotetext{
${ }^{9}$ Muito provavelmente por meio do Cardeal Vissarion que lhe proporcionou o encontro com as obras gregas e o aprendizado da língua (N. A.). 
O sentido moderno de teoria dos números começa com os trabalhos de Pierre Fermat (1601- 1655) com a teoria da divisão por um número primo. No século XVIII Euler avançou mais ainda na teoria dos números: generalizou o resultado de Fermat, criou a teoria geral dos resíduos exponenciais, obteve uma série de resultados sobre a representação de um número na forma de um determinado tipo, investigou uma série de sistemas de equações indeterminadas e obteve resultados em decomposição de um números em parcelas. $\mathrm{O}$ uso de série infinitas e produtos serviu de instrumentos para obtenção de resultados teoriconuméricos. Depois de Euler, uma boa parte dos grandes matemáticos do século XVIII e XIX trabalharam com teoria dos números.

Ao comentar os trabalhos de Gauss (1777-1855), Bukhshtab (1966, p. 10) diz que eles foram importantes para a teoria dos números como um todo e que foi nos trabalhos deste matemático que a teoria das congruências do segundo grau tomou sua forma acabada. Diz também que atualmente (isto é, em 1966) a área ainda se baseia nos resultados expostos no livro Disquisitiones arithmeticae.

No decorrer dos séculos XIX e XX a teoria dos números adquire contornos mais e mais amplos. Mas paremos aqui com o tópico de desenvolvimento da teoria dos números como um todo e voltemos nos concentrar na figura de Tchebyshev.

\title{
A dissertação doutoral e os memorais sobre os números primos
}

Em 1848 escreve Teoria das congruências que defende em 1849 na universidade como dissertação de doutorado. É uma exposição atualizada do que se se tinha feito em teoria dos números até o momento. Com profundo conhecimento dos trabalhos de Euler, Gauss e Legendre encorpora-os num todo e introduz novos elementos. O próprio Tchebyshev escreve na introdução:

\begin{abstract}
"Vemos então que nem os escritos de Legendre e nem os de Gauss apresentam a teoria dos números no formato perfeito no qual ela poderia ser exposta após os desenvolvimentos feitos nesta área pelos estudos feitos por eles mesmos e e muito menos pelos estudos feitos por geômetras posteriores. Por isto na exposição da teoria das congruências eu tive de me orientar não por Legendre ou por Gauss, mas, junto com Legendre e Gauss e muitos outros que trabalharam nesta área da teoria dos números. No entanto, afim de sistematizar os resultados de geômetras que utilizaram abordagens bem distintas, eu tive de mudar a maior parte de suas conclusões. Além do mais, a fim de obter um sistema mais completo, achei necessário desenvolver mais alguns pontos. Assim, na teoria das congruências de primeiro grau eu analiso três casos: quando a congruência tem uma solução, nenhuma solução e várias soluções. Expondo as propriedades das congruencias de ordens superiores, propus em relação a elas vários teoremas de caráter geral além do teorema de Lagrange. Na teoria das formas quadráticas mostro o modo de saber quando duas formas quadráticas de divisores levam a uma forma linear.
\end{abstract}


Além do mais acrescentei três anexos à minha exposição. No primeiro deles..." (Tchebyshev, 1944, p. 14) (tradução nossa, grifo nosso)

O texto de Tchebyshev era uma composição de boa qualidade didática a julgar pelo testemunho de Aleksandr Mikhaílovitch Lyapunov (1857-1918), ex-aluno de Tchebyshev, que diz em conferência pronunciada em 1895:

"O texto Teoria da Congruências serviu durante várias gerações como manual de estudos para os jovens estudantes e até então (isto é, 1895) o texto é considerado a melhor exposição desta parte de teoria dos números". (LYAPUNOV, 1946.) (Tradução nossa, inserção nossa)

Faz todo sentido a afirmação de Lyapunov se lembrarmos que o texto foi escrito quando Tchebyshev foi incumbido de ministrar a disciplina Teoria dos Números pela primeira vez na universidade de São Petersburgo. Além disso, pode-se atestar as repetidas reimpressões do texto ${ }^{10}$.

O texto que Tchebyshev propôs como dissertação de doutorado era composto por:

- uma exposição elaborada, atualizada, corrigida, completada do conhecimento que se tinha da teoria dos números tratada por Euler, Legendre, Gauss e outros contemporâneos e;

- uma parte adicional, resultado de investigação do próprio Tchebyshev contendo tres anexos com os títulos Sobre os resíduos quadráticos, Sobre a determinação de raízes primitivas e Sobre a determinação do número de primos que não ultrapassam dada grandeza.

O terceiro anexo, Sobre a determinação do número de primos que não ultrapassam dada grandeza, Akhiezer ${ }^{11}$ (1946 e 1955) chama de primeiro memorial sobre os números primos. Diz também que o primeiro memorial foi publicado pela academia de ciência de São Petersburgo em 1851 e pela revista de Liouville Journal des Mathématiques Pures et Appliquées ${ }^{12}$ em 1852 (Akhiezer, 1946, p.171)

Tchebyshev escreve ainda outro trabalho importante sobre os números primos ao qual Akhiezer se refere como segundo memorial. Comentando ambos os memoriais ele diz:

\footnotetext{
${ }^{10}$ Sabemos que o texto foi publicado tres vezes em São Petersburgo: a primeira edição foi em 1849, no mesmo ano da defesa, a segunda edição em 1879 e terceira em 1901, edições anteriores à reforma ortográfica de 1918. Foi publicado também em alemão Theorie der Congruenzen, mit Autorisation des Verfassers herausgegeben von H. Shapira, Berlin, 1888; e em italiano Teoria delle congruenze, traduzione com aggiunte e note di Massarini, Roma, 1895.

${ }^{11}$ Naum Ilítch Akhiezer (1901-1980) matemático soviético da área da teoria das funções. No presente artigo ele aparece como um dos principais analisadores e comentadores dos trabalhos de Tchebyshev. Em 1948 foi premiado pela Academia de Ciências da URSS com o prêmio Tchebyshev.

${ }^{12}$ Fundada em 1836 por Joseph Liouville e existente até hoje.
} 
"A fama inicial de P. L. Tchebyshev foi graças aos dois memoriais sobre números primos. $O$ primeiro deles "Sobre a determinação do número de primos" que não ultrapassam dada grandeza surgiu primeiramente como terceiro anexo na Teoria das congruências de que falamos acima. O segundo "Sobre os números primos" foi impresso em 1852 na revista de Liouville. Ambos os trabalhos são dedicados à difícil questão da distribuição dos números primos na sequência dos números naturais, questão esta que vem desde a antiguidade”. (AKHIEZER, 1955, p. 846) (Tradução nossa).

Segundo Akhiezer (1955, p.847) Euler elaborou uma demonstração do teorema sobre a infinitude dos primos na sequência dos naturais. Esta demonstração de Euler junto com a dada por Euclides na antiguidade esgota tudo o que tinha sido rigorosamente demonstrado até o século XIX em relação aos primos na sequência dos naturais. Até mesmo a própria questão da distribuição dos primos não tinha recebido até então um enunciado preciso. Este enunciado surgiu somente em 1808 na segunda edição da Teoria dos Números de Legendre, onde foi proposta uma fórmula empírica para o cálculo aproximado da função $\pi(x)$ para $x$ grande o bastante. Tal fórmula se escrevia como

$$
\pi(x) \approx \frac{x}{\ln (x)-B},
$$

onde $\pi(x)$ indicava o número de primos que não ultrapassam $x$ e B - uma constante que para Legendre era igual a 1,08366. Tal fórmula de fato está em corcordância com a tabela de números primos de 10.000 até 1.000 .000 . No entanto, Tchebyshev acreditava que a fórmula era desprovida de rigor e foi a partir dela (ou de sua crítica) que ele começou o seu primeiro memorial. Trabalhando nesta direção ele foi pioneiro em obter resultados teóricos que relacionam a função $\pi(x)$ com a função $\frac{x}{\ln x}$. Obtém vários resultados importantes nesta direção, inclusive declara corretamente que a fórmula empírica de Legendre não é rigorosa, uma vez que a constante mais adequada seria $B=1$. (AKHIEZER, 1946, p.172)

Descrevendo um pouco mais os dois memoriais de Tchebyshev sobre os números primos podemos dizer, seguindo Deloné $(1947$, p. 42) que o primeiro deles lida com a função-zeta de Euler, com a representação integral desta, com as determinações de suas derivadas simples e logaritmicas. Já o segundo memorial nos deixa admirados ao observarmos o quão elementares são pelas considerações iniciais. Ambos os memoriais denotam a perspicácia e a força de uma mente aguçada presente ao talendo matemática de primeira linha. É com estes dois trabalhos que se inicia a escola de teoria dos números de São Peterburgo. Falaremos mais sobre tal escola na seguinte sessão deste artigo.

Atribuindo um significado histórico ao papel de Tchebyshev, Delone (1947, p. 42) nos diz que os resultados formulados por Tchebyshev sobre teoria geral dos números primos foram os primeiros, depois de Euclides, que versaram de forma precisa sobre a distribuição dos números primos. De fato, Delone continua, é um problema de dificuldade considerável. Após Tchebyshev a série de matemáticos (entre outros, Riemann e Hadamard no final do 
século XIX e início do XX) e mesmo assim, muitas das questões fundamentais continuam em aberto.

\section{Os continuidadores dos estudos em Teoria dos números}

Aleksandr Nikoláievitch Korkin (1837 - 1908) ingressou na Universidade de São Petersburgo em 1854, tornando-se aluno de Bunyakóvsky, Tchebyshev, Sómov, e outros. Terminou o curso universitário e se tornou professor na escola de cadetes em 1858. Em 1860 defendeu a dissertação Determinação de funções arbitrárias em integrais de equações lineares com derivadas parcias que lhe deu o título de mestre. Nesta mesma época Bunyakóvsky deixou a universidade e Korkin foi convidado a ocupar sua vaga. De 1871 a 1877 trabalhou com Zolotariov na teoria das formas quadráticas, o que lhes rendeu reconhecimento na comunidade científica.

Aleksandr Nikolayevitch Korkin lecionou na Universidade de São Peterburgo até 1908, ano de sua morte. (DELONE, 1946, p. 47)

Yégor Ivanovitch Zolotariov (1847 - 1878) nasceu em São Peterburgo. Ao terminar o ginásio dem 1863 começou a assistir aulas como aluno voluntário no curso de matemática. Em 1864 foi incluído como aluno e terminou em1867. Foi aluno de Tchebyshev e Korkin que além das lições, lhe davam conselhos que ajudaram a desenvolver o talento do jovem Zolotariov. Com a idade de 21 anos defendeu a dissertação Uma questão sobre grandezas mínimas e proferiu duas conferências experimentais sendo uma sobre o teorema de Abel e a outra sobre transformação das funções elípticas para a forma normal. Com base na dissertação defendidas e nas duas conferências ele pode ser contratado pela Universidade de São Petersburgo a ministrar o curso de teoria das funções elíptcas para os estudantes de matemática e de cálculo diferencial para os estudantes de ciências naturais.

Em 1870 começa a trabalhar com Korkin sobre os minimum de formas quadráticas positivas. Ao mesmo tempo Zolotariov eleborou teoria dos números algébricos que foi exposta em sua dissertação de doutorado Teoria dos números complexos inteiros com aplicação no cálculo integral. Em 1876 foi indicado Professor da Universidade e no mesmo ano, membro adjunto da Academia de Ciências pela cátedra de matemática aplicada.

Em 26 de junho de 1878, com 31 anos, sofreu um grave acidente de trem vindo a falecer 12 dias depois disso. Porém, neste curto período logrou produzir uma série de trabalhos de fundamental importância. (DELONE, 1946, p. 94)

Andrey Andreyevitch Márkov (1856 - 1922) Estudou no Quinto Ginásio de São Petersburgo e depois disso cursou matemática na Universidade de São Petersburgo formando-se em 1878. Enquanto aluno da universidade foi fortemente influenciado por Tchebyshev, Korkin e Zolotariov, então docentes da universidade. Em 1880 já tinha escrito sua dissertação de mestrado Sobre as formas quadráticas binárias de determinante positivo. Delone (1946, p. 143) faz a seguinte avaliação:

"Este trabalho que foi bem avaliado por Tchebyshev, é um dos pontos altos da escola de teoria dos números de São Petersburgo senão de toda a matemática russa. Basta lembrar das questões na área de aproximações racionais que ocupavam os maiores aritméticos da França e Alemanha 
para entender o quão Markov se adentrou mais longe e mais fundo nesta área. Portanto, não é de admirar que embora o trabalho tenha sido impresso (em francês, nos Matematische Annalen) ele não se tornou um conhecimento comum aos os matemáticos ocidentais e somente nos anos 1910 - 1920 os berlinenses Frobenius e Remak tentaram penetrar na área das ideias construída por Markov. Nos últimos 10 - 15 anos alguns dos maiores aritiméticos ingleses e americanos compreenderam o valor dos resultados obtidos por Markov e tentam desenvolvê-los mais adiante, por enquanto, com sucesso apenas parcial."

Gueórgui Fedocéyevitch Voronoy (1868 - 1908) ingressou na Universidade de São Petersburgo em 1885. A dissertação de mestrado ele defendeu em 1894 sendo logo após nomeado Proféssor da Universidade de Varsóvia. Em Varsóvia ele buscou elaborar um algoritmo que o permitisse resolver no campo cúbico as mesmas questões que o algoritmo das frações contínuas resolve no campo quadrático. Este trabalho foi defendido em 1897 com o título Sobre uma generalização do algoritmo de frações contínuas na Universidade de São Petersburgo.

Ivan Matvéievitch Vinográdov (1891 - 1983) foi aluno da faculdade fisico-matemática da Universidade de São Petersburgo formando-se em 1914. Dedicou seu trabalho à teoria analítica dos números. Esta área que surgiu no século XIX alcançou ultimanente um grande desenvolvimento. A temática foi trabalhada fora da Russia por Hardy, Littlewood e van der Korput , todos eles seguindo os passos indicados por matemáticos anteriores, isto é, aplicando métodos da análise (em especial, a teoria das funções de variável complexa) às funções analíticas que de um modo ou de outro estão relacionadas às propriedades dos números inteiros. Vinográdov em 1937 encontrou uma solução para o problema ternário de Goldbach ${ }^{13}$ para números suficientemente grandes (ou seja, encontrou uma solução parcial). O teorema foi demonstrado completamente somente em 2013 por Harald Helfgott.

\section{Conclusão}

A academia de ciências de São Petersburgo foi criada por um decreto do imperador Pedro I (Predro, o Grande) em 24 de janeiro de 1724. O imperador foi aconselhado a trazer da Europa Ocidental quadros de primeira linha para formar o corpo de pesquisadores da academia. E assim foram convidados a vir morar e trabalhar na Rússia homens de ciência já reconhecidos em suas respectivas áreas de atuação. Os primeiros matemáticos a chegar foram escolhidos a dedo: Jacob German, que fora aluno de Jacob Bernulli; Daniel e Nicolaus, ambos filhos de Johann Bernulli e Christian Goldbach. Além de nomes já conhecidos internacionalmente, o convite também se fazia a jovens promissores e foi nesta condição que em 1727 chegou a São Petersburgo o jovem Leonhard Euler, então com 20 anos de idade e que se tornou um dos matemáticos mais produtivos de todos os tempos.

\footnotetext{
${ }^{13}$ Em 1742 o matemático Christian Goldbach em carta a Leonhard Euler levantou a seguinte
} hipótese: todo número ímpar maior que nove é a soma de tres números.

RBHM, Vol. 16, no 32, p. 19-31, 2016 
Euler permaneceu em São Petersburgo até 1741 quando pediu dispensa dos seus serviços e seguiu para a Europa Ocidental. Trabalhou por 25 anos na Prússia mas manteve laços com a academia de São Petersburgo por meio de publicações. Retornou a São Peterburgo em 1766 e aí permaneceu até sua morte em 1783.

Após a morte de Euler a academia de São Peterburgo perdeu seu brilho matemático. (GNEDENKO, 1946, p. 83). Mas sabemos que algum tempo após isto a Rússia já tinha matemáticos da grandeza Lobatchevsky (1792 - 1856) Bunyakovsky (1804 - 1889) e Tchebyshev (1821-1894). Este hiato que vai da morte de Euler até o despontar dos matemáticos russos é um outro estudo que não cabe no escopo do presente artigo.

Sabe-se que uma parte da enorme produção de Euler é sobre teoria dos números. No entanto, Gnedenko (1946, p. 83) diz que Euler teve oito alunos que mais tarde se tornaram membros da academia de São Petersburgo, mas, que nenhum deles foi um substituto à altura de seu mestre em produção matemática. Quando Tchebyshev começou a trabalhar em com a teoria dos números ele partiu das contribuições de Euler, Lagrange, Abel e Gauss. Tchebyshev, por sua vez, deixou continuadores de sua obra, o que nos permite dizer que com ele iniciou-se a escola russa em teoria dos números.

\section{Bibliografia}

AKHIEZER, Naum Ilítch.1955. P. L. Tchebyshev y ievo naútchnoe nasledie (P. L. Tchebyshev e seu legado científico). In: VINOGRADOV, Ivan Matvéievitch (Red.). Ízbranie Trudí P. L. Tchebysheva (Obras escolhida de P. L. Tchebishev). Moscou, Akademya Naúk URSS. 930 p. (Em russo)

BERNSHTEIN, S. (Red.). 1944. Pôlnoie Sobrânie Sotchinêniy - 5 T. (Obras completas de P. L. Tchebyshev em 5 tomos). Academya Naúk URSS, Moskvá-Leningrad, 1944. Tomo I, 343 p. (Em russo)

BUKHSHTAB, Aleksándr Adólfovitch. 1966. Teorya Tchícel (Teoria dos Números). Izdátelstvo Prosvechênye, Moscou. (Em russo).

DELONE, Nikolai Boríssovitch. 1947. Peterburgskaya shkola teorii tchícel (A escola de Teoria dos Números de São Petersburgo). Akademya Naúk URSS. Naútchno-populyarnaya séria. Moskvá-Leningrad. (Em russo)

GNEDENKO B. V. 1946. Otcherki po istorii matematiki v Rossii. OGIZ, Moskba Leningrad (Em russo).

LYAPUNOV, A. M. 1946. Pafnútiy Lvóvitch Tchebyshev. In: OGIZ. 1946. P. L. Tchebyshev - Ízbranie Matematítcheskie Trudí. (P. L. Tchebyshev - Obras matemáticas escolhidas) Série Clássicos das Ciências Naturais, Izdádelstivo OGIZ Moscou - Leningrado. Pp. 9-21. (Em russo)

OGIZ. 1946. P. L. Tchebyshev - Ízbranie Matematítcheskie Trudí. (P. L. Tchebyshev - Obras matemáticas escolhidas) Série Clássicos das Ciências Naturais, Izdádelstvo OGIZ Moscou Leningrado. 200 p. (Em russo)

PRÚDNIKOV, Vassíliy Efímovitch. 1976. Pafnútiy Lvóvitch Tchebyshev 1821-1894. Editado pela Akademya Naúk URSS. Série científico-biográfica. Editora Naúka-Sessão Leningrado. Leningrado. (Em russo). 
TCHEBYSHEV, P. L. (1944). Teorya Sravnêniy. In: BERNSHTEIN, S. (Red.). Obras completas de P. L. Tchebyshev. Academya Naúk URSS, Moskvá-Leningrad, 1944. Tomo I, pp. 10 - 172. (Em russo)

VINOGRADOV, Ivan Matvéievitch (Red.).1955. Ízbranie Trudí P. L. Tchebysheva (Obras escolhida de P. L. Tchebishev). Moscou, Akademya Naúk URSS. 930 p. (Em russo)

\author{
Bernadete Morey \\ Departamento de Matemática - UFRN - campus de \\ Natal - Brasil \\ E-mail: bernadetemorey@gmail.com \\ Adgam Sultanov \\ Departamento de Matemática - Universidade \\ Estatal de Penza - Penza - Rússia \\ E-mail: tyapin_nikita@mail.ru
}

